\title{
Centering and marginalization in introductory university physics courses
}

\author{
Rachel E. Scherr \\ School of STEM, University of Washington Bothell, 18115 Campus Way NE, Bothell, WA, 98011 \\ W. Tali Hairston \\ Equitable Development LLC, Seattle, WA \\ Sarah B. McKagan \\ Alder Science Education Association, Seattle, WA
}

\begin{abstract}
Research-based instructional strategies in physics promote active participation in collaborative activities as a primary means for students to construct understanding. This emphasis is in line with situated learning theory, in which learning is indicated by a student's increasing centrality in a community. In both perspectives, to learn more is to engage more centrally: to start discussions, conduct experiments, write on the board, decide when a question has been answered, and so on. In a study of small-group collaborative learning activities in introductory physics classrooms at three different universities, we observe that as students engage with one another and with instructors, they are not only negotiating physics concepts, but also negotiating social positioning. Some students are centered (and their contributions are valued), while others are marginalized (and their contributions are neglected). The aim of this research is to become conscious of how centering and marginalization shape the way physics is taught and learned.
\end{abstract}




\section{INTRODUCTION}

Research-based instructional strategies in physics promote active participation in collaborative activities as a primary means for students to construct understanding [1] [3]. This emphasis is in line with situated learning theory (SLT), in which learning is indicated by a student's increasing centrality in a community [4]-[6]. This centrality may be physical, as when everyone in a group orients to a specific person, or symbolic, as when everyone in a group orients to an idea that is considered to be at the heart of some matter of consequence. People in a central position have access to full participation in the activity; in a small-group collaborative physics activity, for example, they start discussions, conduct experiments, write on the board, or decide when a question has been answered. Others, whose position is more peripheral, may be physically located on the margins of the activity or may participate less fully in it. In addition to indicating learning, movement from periphery to center is also associated with identity development: as participants move toward full participation in a physics activity, they increasingly identify as physics students. Overall, this model contrasts with others in which learning is seen as an individual cognitive achievement and knowledge is a psychological or volitional reality of an individual.

In a study of small-group collaborative learning activities in introductory physics classrooms at four different universities, we observe that discussions of physics content are also negotiations of social position, and may not reflect the learning of physics concepts in a cognitive sense [7]. Some students are centered (and their contributions are valued), while others are marginalized (and their contributions are neglected). The aim of this research is to become conscious of how centering and marginalization shape the way physics is taught and learned. Our contribution is to add critical consciousness to the SLT perspective, in a physics learning context.

\section{THEORETICAL FRAMEWORK}

Situated learning theory is a framework that defines learning in terms of the practices of a community [4]-[6]. In this framework, learners move from legitimate peripheral participation into more central positions as they are acculturated through knowledge acquisition and identity development [4]-[6], [8], [9]. SLT is often used to describe learning in the community or the workplace, but also provides a framework for analyzing school learning [10]. SLT's emphasis on centrality aligns with common values in physics education research: in both perspectives, a key component of learning is increasingly engaged participation in learning activities (e.g., starting discussions, conducting experiments, writing on the board, deciding when a question has been answered). In physics classrooms as in other contexts, a participant's mastery of the situated practices, knowledge, and skills of the community determines centrality and sustains the practices of the community [4][6].

SLT has been criticized by critical scholars such as Esmonde and Booker for paying little attention to the power relationships that are negotiated in communities of practice, for not considering race, gender, or other social identities to be barriers to participation, and for treating students who remain peripheral as failing to identify as expert learners, regardless of social identity [8]-[10]. Critical scholars have pointed out that power relationships are key to learning in the SLT model because access to the cultural system, and its associated identity development, is determined by those positioned at the center [8]-[11]. Power relationships are not static, but negotiated [10]: power is (or is not) shared by the expert with the novice in a legitimate negotiation of shared identity [8], [12].

Critiques of SLT argue that full participation within a community of practice, such as a classroom, creates inequitable opportunities when power relationships and member identity are negotiated inconsistently, often based on sociocultural identity [10], [13]-[15]. For example, a learner's sociocultural identity may conflict with their community of practice identity, thus preventing full participation: e.g., a women of color may be questioned because her body does not conform to prevalent images of the "ordinary" white male physicist. [16]-[19]. Social relations ascribed by the broader sociocultural context may not value the membership of diverse others, in which case the learning of those diverse others will be limited [12]. For example, in a year-long ethnographic study of female Japanese graduate students in a Canadian university, students negotiated and constructed identity in an attempt to gain a more central position in the academic community of practice: the movement to a more central position required identity formation and learning, but the students did not always get access to necessary cultural resources [12]. Identity formation as a full participating member therefore may not be accessible to all students regardless of the learner's willingness to participate. In such circumstances, structural inequities and epistemological hierarchy are reinforced [10], [11].

In physics, which has among the lowest representation of women, gender-diverse people, and people of color of any academic discipline [20], [21], theories of learning need to address the lack of mutuality that people in underrepresented groups experience. When students from underrepresented groups do not move from peripheral to central positions in a learning community, it may be because the community of practice does not allow them to learn. Without a critical lens to account for the situatedness of race, gender, or even patriarchy within the power relations, physics education research risks reproducing structural inequities. Acculturation with no assessment of the power relationship, 
or attention to sociocultural identities, ensures learning as identity construction is a type of colonization [10], [11].

In this study, we analyze physics learning interactions from a SLT perspective that includes critical consciousness [8]-[10], [16], [22], [23]. We characterize interactions among students in terms of centrality and marginalization, and attend to the sociocultural identities that may be barriers to participation.

\section{METHODS}

We video recorded 1-2 weeks of introductory physics classes at three different universities, totaling approximately 10-20 hours of video for each site. The project team and the study site leader selected the classes to be videotaped by mutual agreement, with attention to classes that feature (1) frequent or in-depth interactions among students and between students and instructors and (2) racial, ethnic, or gender diversity. From the body of video data for each site, the project selected sequences of events in which centering and/or marginalization was visibly sustained or contested. Evidence of centrality comes from both non-verbal cues e.g., multiple people in the video turn toward a speaker - and discourse analytic markers indicating that an idea or person is being elevated - e.g., "that's a great idea" [7], [24]-[26]. Such interactions, when they occur repeatedly, consistently, or in a sustained manner over time, indicate that someone is being sustained in a central position. We also selected episodes in which centrality was contested: when an otherwise peripheral participant tried to gain the floor [7], contribute knowledge, or direct the group's activity. Five to eight episodes were selected from each university. To inform and triangulate the analysis of selected classroom video episodes, the project team typically conducted 2-3 stimulated recall interviews [27]-[29] per site, in which faculty or students who appear in a classroom video episode offer their perspective on those specific events.

\section{EXAMPLE OF CENTERING AND MARGINALIZATION}

The following episode takes place in an introductory physics class at a small private university in the western United States. The class uses evidence-based practices including tutorials [2] to support small-group collaborative activities. The project videotaped two weeks in the middle of the autumn quarter course in mechanics, recording two out of six groups four times a week, for one to two hours each class session.

In the following episode, students are working on a tutorial activity about acceleration in one dimension. The students, pseudonymed Andy, Bill, Cindy, and Dan, sit together for each class session (see Fig. 1). Andy, Bill, and Dan present as white men and Cindy presents as a white woman. We selected this group particularly because all of the students in it present as white, which is the dominant racial culture represented in physics: as such, it tends to be treated as the norm and therefore ignored. By analyzing the interactions of white students, we analyze a case of the white culture that is pervasive in physics.

Overall, the positioning for this group is highly stable: the same individuals are centered or marginalized to approximately the same extent in all of the class sessions observed for the study. In SLT terms, the legitimate peripheral participants are kept at the periphery, and thus reified as novices.

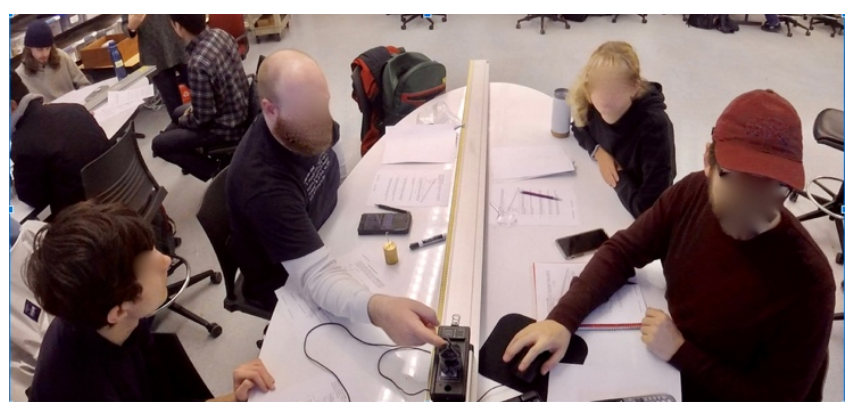

FIG. 1. Clockwise from bottom left: Andy, Bill, Cindy, and Dan.

\section{A. Episode}

In this episode, Andy, Bill, Cindy, and Dan are predicting how the acceleration of a cart on a ramp will change when they change the mass of the cart. The cart, ramp, and motion sensor are set up at their table so that they can run experiments to test their predictions. Andy asks if the weight of the cart is $700 \mathrm{~g}$, and Bill says it is $0.8 \mathrm{~kg}$. Bill sets up the cart, Dan starts the motion detector, and Cindy stops the cart. Andy states that the acceleration would decrease when the mass is decreased, according to Newton's Second Law (in which he presumes, incorrectly, that the net force is constant):

Andy: So the mass of the base cart is 700 grams, right?

Bill: Uh, it's about 0.8 kilograms.

[12 seconds in which Bill arranges the equipment]

Bill: You ready to test our prediction?

Dan: Oh, yeah, sure.

Andy: Wouldn't the acceleration decrease?

Dan: We don't have enough significant figures.

Cindy: Yah.

Andy: $\mathrm{F}=\mathrm{ma} . .$. Why would acceleration increase? Acceleration should decrease. $\mathrm{F}=$ ma so assuming that force is constant...

Amidst some talk about running the experiment, Bill responds to Andy with, "Oh. Right. No. The acceleration shouldn't do anything. Thank you," seeming to agree with Andy, while also seeming to state that the acceleration would stay the same (which is not what Andy said). Andy next says, "Acceleration is still just due to gravity," now seeming to agree with Bill that the acceleration of the cart is independent of its mass.

Once Dan has finished assisting with the experiment, he and Cindy do not attend to Andy and Bill's interchange. 
Instead, they focus on a graph on the computer screen, which likely represents the output of the motion sensor. Dan has the mouse and manipulates the graph, appearing unsatisfied with the result. Cindy makes a technical suggestion ("Put a highlighter on it"), and then says "Hello?", suggesting she feels Dan is not listening to her. She requests the mouse by wiggling her fingers. Dan throws up his hands, releasing the mouse to her, and she uses the mouse to make an adjustment to the graph (see Fig. 2). As Cindy gains the mouse, Andy and Bill look down at their papers and write.

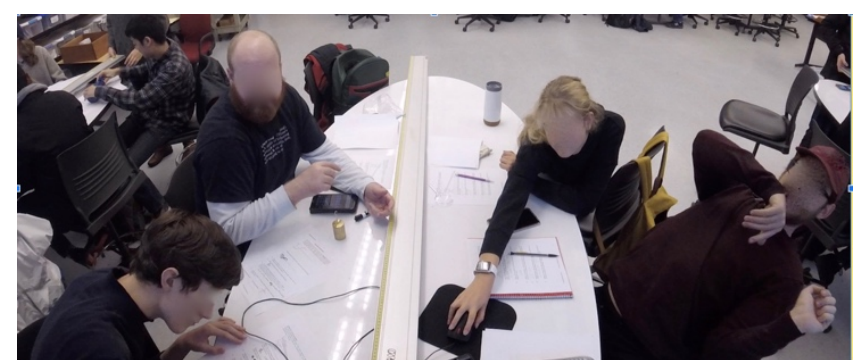

FIG. 2. Cindy reaches for the mouse.

\section{B. Centering and marginalization}

This episode shows several behavioral patterns that we observe to be typical for this group over the two-week period of video recording. One student, Bill, is centered: he directs the group's action (such as deciding when to run the experiment) and makes intellectual decisions for the group (what the mass of the cart is, and whether the acceleration is independent of mass). In a stimulated recall interview, Andy affirms Bill's centering as typical: he says, "Usually I deferred to his opinions and his reasoning because it was usually right." Andy described this episode as unusual in that Andy made a bid to correct Bill's reasoning; Andy says he "phrased it hesitantly because [Bill is] really smart, he's in his third year doing a master's in physics, just retaking this class because he transferred," suggesting that Bill has unusually high expertise for an introductory physics class. Andy adds that "usually when I ask something like that, about half the time I'm probably wrong, if I'm trying to correct him, because he's really good at physics." In other words, Bill is centered partly because other students attribute academic merit to him. In this episode, Andy makes a bid to move toward the center (by "trying to correct" Bill), but perceives this action as unusual (thus he "phrased it hesitantly"). In the end, Bill's centralized position is sustained: Andy's contribution is absorbed and changed into a different statement made by Bill.

Dan appears close to the center occupied by Bill in this episode: he carries out Bill's suggestion to run the experiment, and he takes the lead in manipulating the graphical output of the motion sensor (he initially holds the mouse). Cindy makes a bid to move toward the center by making a technical suggestion about how to manipulate the graph ("Put a highlighter on it"), but her move is ignored.
Cindy's "Hello?" shows her own recognition that she is on the periphery of the action: that others are not listening to her, and that if she is going to be heard, she needs to take more action. She does so by requesting the mouse; her move is treated as unusual (Dan throws up his hands to release the mouse to her, as if doing so was a major event). None of the other group members attend to her manipulation of the graph. In an interview, Andy described Cindy's low profile as typical for their group: he says Cindy is "usually a little more quiet. She and Dan are good friends and she mostly defers to what he says."

Overall, two students in this group are centered: primarily Bill, and secondarily Dan. In this episode, Andy and Cindy both make bids for centrality: these moves are seen as non-normative (Andy describes his move as rare, and Dan throws up his hands), and do not disrupt the overall structure of the group. Situated learning theory would pose Bill and Dan as being more expert-like and having a secure identity as physics learners, as evidenced by their central positions. Physics education research would see Bill and Dan as successful learners in that they are actively engaged with the material: they are "hands-on," they direct action, they are visibly engaged in the instructional activities, they share their ideas, and they pursue answers to physics questions. Andy and Cindy, meanwhile, would be understood by SLT to be legitimate peripheral participants in the group: they engage in simpler, lower-risk tasks, such as asking about the mass of the cart or making suggestions about the computer display. Overall, SLT and PER would tend to judge Bill and Dan as more successful learners than Andy and Cindy, in this episode.

\section{Critical perspective}

Missing from the above analysis is the critical perspective that to move to the center in a group is to negotiate power, sometimes inequitably. Bill and Dan appear to be white men, a social identity that is greatly overrepresented in physics at all levels [20], and display certain characteristics that U.S. culture typically associates with white masculine behavior, including control, independence, and decisiveness [30]. Although Andy also presents as a white man, he does not exhibit many characteristics typically associated with white masculine behavior. Bill's decisionmaking (about the mass of the cart, the timing of the experiment, and the group's prediction) has the effect of marginalizing Andy: Andy's "hesitant" suggestions are not taken up by the team, and are even rendered less visible by Bill's discourse (e.g., when Bill reframes Andy's prediction as something other than what Andy said). In other words, the centering of Bill produces the marginalization of Andy.

Meanwhile, on the other side of the table, Dan and Cindy's interaction typifies some patterns of gendered interaction in physics, including men getting more attention and more access to equipment [14], [15], [18], [31]-[33]. For 
example, Dan's possession of the mouse makes the mouse initially inaccessible to Cindy. When Cindy obtains the mouse, she does not gain the group's attention: at that moment, Andy and Bill look away from the computer display, down to their individual worksheets. This marginalization, though contested (e.g., by Cindy obtaining the mouse), is not significantly disrupted during this episode.

Overall, Andy and Cindy are not only on the periphery, but are kept to the periphery with behavioral patterns that typify racialized and gendered interactions.

\section{DISCUSSION}

This analysis describes a physics learning interaction from a SLT perspective that includes critical consciousness [8]-[10], [16], [22], [23]. Interactions among students are characterized in terms of centrality and marginalization, with attention to the sociocultural identities that shape participation. We argue, in line with work by Esmonde and Booker, that research using SLT must attend to the power structures associated with sociocultural identities in order to properly characterize students' learning [10]. The power structures and discourse norms of the dominant (white masculine) culture act on students' social identities like a kind of gravity, producing centering and marginalization and thereby distorting opportunities for learning. Future work will examine these dynamics in greater depth, with other groups (including mixed-race groups), and with instructor interactions.

\section{ACKNOWLEDGMENTS}

We are grateful to Amy D. Robertson for significant contributions to this analysis. This work was supported in part by the National Science Foundation under grant number 1760761 .
[1] E. Brewe, "Modeling theory applied: Modeling Instruction in introductory physics," Am. J. Phys., vol. 76, no. 12, pp. 11551160, Nov. 2008, doi: 10.1119/1.2983148.

[2] P. S. Shaffer and McDermott, L. C., Tutorials in Introductory Physics Updated Preliminary Second Edition 2011-2012. New York? Pearson, 2012.

[3] C. H. Crouch and E. Mazur, "Peer Instruction: Ten years of experience and results,” Am. J. Phys., vol. 69, no. 9, pp. 970977, Aug. 2001, doi: 10.1119/1.1374249.

[4] J. Lave and E. Wenger, Situated Learning: Legitimate Peripheral Participation, 1st edition. Cambridge England; New York: Cambridge University Press, 1991.

[5] J. Lave, "Situating learning in communities of practice," in Perspectives on socially shared cognition, Washington, DC, US: American Psychological Association, 1991, pp. 63-82.

[6] E. Wenger, Communities of practice: Learning, meaning, and identity. New York, NY, US: Cambridge University Press, 1998, pp. xv, 318.

[7] R. A. Engle, J. M. Langer-Osuna, and M. M. de Royston, "Toward a Model of Influence in Persuasive Discussions: Negotiating Quality, Authority, Privilege, and Access Within a Student-Led Argument," J. Learn. Sci., vol. 23, no. 2, pp. 245-268, Apr. 2014, doi: 10.1080/10508406.2014.883979.

[8] A. Contu and H. Willmott, "Re-Embedding Situatedness: The Importance of Power Relations in Learning Theory," Organ. Sci., vol. 14, no. 3, pp. 283-296, Jun. 2003, doi: 10.1287/orsc.14.3.283.15167.

[9] J. Curnow and J. R. Chan, "Becoming an 'Expert': Gendered Positioning, Praise, and Participation in an Activist Community," Jul. 2016, Accessed: May 18, 2020. [Online]. Available: https://repository.isls.org//handle/1/110.

[10] I. Esmonde and A. N. Booker, Power and Privilege in the Learning Sciences: Critical and Sociocultural Theories of Learning. Taylor \& Francis, 2016.

[11] M. Bang and S. Vossoughi, "Participatory Design Research and Educational Justice: Studying Learning and Relations Within Social Change Making," Cogn. Instr., vol. 34, no. 3, pp. 173-193, Jul. 2016, doi: 10.1080/07370008.2016.1181879.

[12] M. Haneda, "Classrooms as Communities of Practice: A Reevaluation,” TESOL Q., vol. 40, no. 4, pp. 807-817, 2006, doi: $10.2307 / 40264309$.

[13] J. Hughes, N. Jewson, and L. Unwin, Communities of Practice: Critical Perspectives. Routledge, 2013.

[14] A. J. Gonsalves, A. Danielsson, and H. Pettersson, "Masculinities and experimental practices in physics: The view from three case studies," Phys. Rev. Phys. Educ. Res., vol. 12, no. 2, p. 020120, Aug. 2016, doi: 10.1103/PhysRevPhysEducRes.12.020120.

[15] R. S. Barthelemy, M. McCormick, and C. Henderson, "Gender discrimination in physics and astronomy: Graduate student experiences of sexism and gender microaggressions," Phys. Rev. Phys. Educ. Res., vol. 12, no. 2, p. 020119, Aug. 2016, doi: 10.1103/PhysRevPhysEducRes.12.020119.

[16] S. Hyater-Adams, C. Fracchiolla, T. Williams, N. Finkelstein, and K. Hinko, "Deconstructing Black physics identity: Linking individual and social constructs using the critical physics identity framework," Phys. Rev. Phys. Educ. Res., vol. 15, no. 2, p. 020115, Aug. 2019, doi: 10.1103/PhysRevPhysEducRes.15.020115.

[17] M. Ong, "Body Projects of Young Women of Color in Physics: Intersections of Gender, Race, and Science," Soc. Probl., vol. 52, no. 4, pp. 593-617, Nov. 2005, doi: 10.1525/sp.2005.52.4.593.

[18] M. Ong, C. Wright, L. Espinosa, and G. Orfield, "Inside the Double Bind: A Synthesis of Empirical Research on Undergraduate and Graduate Women of Color in Science, Technology, Engineering, and Mathematics," Harv. Educ. Rev., vol. 81, no. 2, pp. 172-209, Jun. 2011, doi: 10.17763/haer.81.2.t022245n7x4752v2.

[19] K. Rosa and F. M. Mensah, "Educational pathways of Black women physicists: Stories of experiencing and overcoming obstacles in life,” Phys. Rev. Phys. Educ. Res., vol. 12, no. 2, 
p. 020113, Aug. 2016, doi: 10.1103/PhysRevPhysEducRes.12.020113.

[20] "The Integrated Postsecondary Education Data System." https://nces.ed.gov/ipeds/use-the-data (accessed Jun. 06, 2019).

[21] L. J. Sax, K. J. Lehman, R. S. Barthelemy, and G. Lim, "Women in physics: A comparison to science, technology, engineering, and math education over four decades," Phys. Rev. Phys. Educ. Res., vol. 12, no. 2, p. 020108, Aug. 2016, doi: 10.1103/PhysRevPhysEducRes.12.020108.

[22] P. Freire and D. Macedo, Pedagogy of the Oppressed, 30th Anniversary Edition, 30th Anniversary edition. New York: Continuum, 2000.

[23] S. Hyater-Adams, C. Fracchiolla, N. Finkelstein, and K. Hinko, "Critical look at physics identity: An operationalized framework for examining race and physics identity," Phys. Rev. Phys. Educ. Res., vol. 14, no. 1, p. 010132, Jun. 2018, doi: 10.1103/PhysRevPhysEducRes. 14.010132.

[24] C. Goodwin, "Action and embodiment within situated human interaction," J. Pragmat., vol. 32, no. 10, pp. 1489-1522, Sep. 2000, doi: 10.1016/S0378-2166(99)00096-X.

[25] A. Kendon, Conducting Interaction: Patterns of Behavior in Focused Encounters. CUP Archive, 1990.

[26] S. J. Derry et al., "Conducting Video Research in the Learning Sciences: Guidance on Selection, Analysis, Technology, and Ethics,” J. Learn. Sci., vol. 19, no. 1, pp. 3-53, Jan. 2010, doi: 10.1080/10508400903452884.

[27] J. Calderhead, "Stimulated Recall: A Method for Research on Teaching,” Br. J. Educ. Psychol., vol. 51, no. 2, pp. 211-17, Jun. 1981.

[28] N. P. Dempsey, "Stimulated Recall Interviews in Ethnography," Qual. Sociol., vol. 33, no. 3, pp. 349-367, Sep. 2010, doi: 10.1007/s11133-010-9157-x.

[29] J. Lyle, "Stimulated Recall: A Report on Its Use in Naturalistic Research,” Br. Educ. Res. J., vol. 29, no. 6, pp. 861-878, 2003.

[30] J. W. Scott, "Gender: A Useful Category of Historical Analysis,” Am. Hist. Rev., vol. 91, no. 5, pp. 1053-1075, 1986, doi: $10.2307 / 1864376$.

[31] D. Doucette and C. Singh, "Why Are There So Few Women in Physics? Reflections on the Experiences of Two Women," Phys. Teach., vol. 58, no. 5, pp. 297-300, May 2020, doi: 10.1119/1.5145518.

[32] S. Herrera, I. A. Mohamed, and A. R. Daane, "Physics from an Underrepresented Lens: What I Wish Others Knew," Phys. Teach., vol. 58, no. 5, pp. 294-296, May 2020, doi: 10.1119/1.5145517.

[33] A. T. Danielsson, "Exploring woman university physics students 'doing gender' and 'doing physics,"' Gend. Educ., vol. 24, no. 1, pp. 25-39, Jan. 2012, doi: 10.1080/09540253.2011.565040. 